\title{
UN ANÁLISIS ESTRUCTURAL PARA LA INFLACIÓN EN ESTADOS UNIDOS
}

\section{Luis Efrén Yáñez Villegas ${ }^{1}$}

\section{Yessica Guadalupe Bautista Baeza ${ }^{2}$}

\section{Resumen}

La inflación es, en todas las economías, un termómetro de la actividad económica, su estabilidad financiera; así como uno de los indicadores más importantes del poder adquisitivo de un país. En este sentido, el estudio de los determinantes de la inflación es crucial para prevenir choques inflacionarios a corto y mediano plazo. Desde el siglo pasado se han vinculado las variables monetarias como únicas causales de la inflación, excluyendo por completo al enfoque inflacionario estructural. El propósito de este artículo es indagar en la determinación de la inflación por orígenes estructurales. La hipótesis central propone al precio de la gasolina regular como un causal directo de la inflación. Se estudian las diferentes teorías inflacionarias y se examina la transferencia del precio del petróleo a la inflación de Estados Unidos (una economía con tasas de inflación ultra bajas). Para corroborar dicha hipótesis se realiza un modelo econométrico Autorregressive Movil Average Exogenous (ARMAX). Este establece a los precios de la gasolina y los rezagos de la inflación como determinantes inflacionarios. Finalmente, se pronostican datos mensuales para la inflación de 2019 .

Palabras clave: causales inflacionarias, determinantes estructurales, gasolina media.

1 Alumno de la licenciatura de Economía, de la Universidad Autónoma Metropolitana, Unidad Iztapalapa, correo electrónico: efreny71@gmail.com.

2 Licenciada en Economía, por la Universidad Autónoma Metropolitana, Unidad Iztapalapa, correo electrónico: yessicabautistabaeza@outlook.es. El presente artículo forma parte de un trabajo más amplio que se hizo acreedor al premio a la investigación para estudiantes en el área de Economía 2019 de la UAM-I, contando con la asesoría del profesor Roberto Gutiérrez Rodríguez y las sugerencias del alumno doctorante en Economía Social Luis Amado Sánchez. Además, el trabajo participó en el evento anual de investigación Reto Banxico 2019.

* Fecha de recepción: 30/03/2020. Fecha de aceptación: 11/05/2020. 


\begin{abstract}
Inflation in all economies is a thermometer of economic activity, its financial stability and one of the most important parameters of the purchasing power of a country. In this sense, the study of the direct determinants of inflation is crucial to prevent inflationary shocks in the short and medium term. Since the last century, monetary variables have been linked as sole causes of inflation, completely excluding the structural inflationary approach. The purpose of this article is to investigate in the determination of inflation by structural origins, the central hypothesis being that the price of regular gasoline as a direct cause of inflation. The different inflationary theories are studied and the transfer of the oil price to the inflation of the United States (an economy with ultra-low inflation rates) is examined. To corroborate this hypothesis, an Autorregressive Movil Average Exogenous (ARMAX) econometric model is performed, which links gasoline prices and inflation lags as inflationary determinants. Finally, monthly data is forecast for 2019 inflation.
\end{abstract}

Keys words: inflationary causes, structural determinants, average gasoline.

\title{
1. Introducción
}

La inflación es en esencia, el aumento sostenido de los precios de una canasta de bienes y servicios representativa de un periodo de tiempo (generalmente un año). La naturaleza de esta variable permite conocer el estado de salud de una economía al ser uno de los indicadores del consumo de sus agentes económicos y del poder adquisitivo de su moneda.

Por esta razón, el estudio de los determinantes inflacionarios es clave para predecir cambios en el comportamiento de las principales variables macroeconómicas. Hasta ahora, se ha intentado explicar el comportamiento de la inflación desde la vertiente de política monetaria (que incluye variables tales como la tasa de interés objetivo, el tipo de cambio y la oferta monetaria). Sin embargo, muy pocos estudios económicos han intentado explicar a las variaciones inflacionarias como una 
consecuencia de los desajustes en las variables estructurales, entre ellas la oferta y demanda de hidrocarburos, especialmente el petróleo.

El presente artículo estudia la inflación bajo un enfoque híbrido, donde la principal hipótesis subyace en proponer a los precios de la gasolina como un determinante esencial en la formación de precios. También, se plantean a los rezagos inflacionarios como causales de la misma variable, es decir, que los valores rezagados de la inflación repercuten en los valores actuales.

La estructura de la investigación sigue tres ejes centrales: 1) se describe el debate de las causales inflacionarias y se exponen las principales teorías al respecto; 2) se explica el proceso de transferencia de precios del petróleo a los movimientos inflacionarios, destacando el caso de Estados Unidos, una economía con tasas de inflación extra bajas. Adicionalmente, se añade un análisis que vincula a las principales variables macroeconómicas y financieras con la inflación de dicho país; 3) se realiza un modelo econométrico ARMAX (Autorregressive Movil Average Exogenous) para probar la hipótesis antes planteada y se desprenden pronósticos mensuales para 2019.

Concluimos que las fluctuaciones del precio de la gasolina tienen un efecto limitado, pero duradero, sobre la inflación subyacente; $y$, por tanto, sobre la inflación en Estados Unidos.

\section{El debate sobre las causas de la inflación}

Existe cierta ambigüedad en establecer, a ciencia cierta, cuál es el origen de un proceso inflacionario ya que sus causas son muy distintas de una economía a otra. Por ejemplo, en una economía donde se impuso un régimen inflacionario, no es necesario preguntarse el porqué del cambio en precios (CEPAL, 1986), o en una economía con continuos choques políticos que cierra sus fronteras y desabastece sus principales bienes de consumo. Los cambios en las principales variables monetarias (tipo de cambio, tasas de interés y oferta de dinero) son vistos como los posibles determinantes de la inflación (Heath, 2012) por la mayoría de los bancos centrales del mundo. Sin embargo, considerarlos como a es- 
tos como los causales únicos de la inflación puede resultar ciertamente ambiguo. Debido a que sus oscilaciones son de índole exógena (una elevación del tipo de interés mundial provoca una salida de capitales, que alteran la inversión y el consumo de un país, por tanto, sus precios) (CEPAL, 1986).

Por ende, se corre el riesgo de dar explicaciones exorbitantemente cerradas o simplistas o llevar la discusión de los determinantes inflacionarios a afirmaciones medianamente precisas (CEPAL, 1986). En consecuencia, debe tratarse al cambio en precios desde el proceso que origina la inflación, lo cual implica evaluar el precio de los bienes de consumo que están representados en la canasta básica del nivel de precios.

A continuación, se presentan las principales teorías sobre los causales de la inflación:

\section{a) Las teorías monetario-fiscales}

De acuerdo con Friedman, la inflación es siempre y en todo lugar un fenómeno monetario (Friedman, 1968). El monetarismo, a diferencia de los esquemas de la teoría cuantitativa simple (donde la determinación de la inflación era un causal de los esquemas de capacidad), intenta explicar a la inflación por los continuos choques en su oferta monetaria ${ }^{3}$, ingreso nominal, tipo de cambio y tasa de interés (CEPAL, 1986). Estos choques podían impactar directamente en el periodo activo o como rezagos de un año.

3 La teoría tiene dos componentes: la asociación entre dinero y precios y la determinación de la masa monetaria. En respuesta al primer componente, se estudia al tipo de cambio según su vínculo con el interior o exterior: una economía cerrada depende de su cantidad de dinero en circulación; mientras una abierta, el arbitraje entre compras y ventas del exterior fija los precios internacionales y el tipo de cambio. En cuanto al segundo componente, la masa monetaria es consecuencia de la paridad de poder adquisitivo. En una economía con tipo de cambio flotante, la masa monetaria actúa positivamente sobre los precios (CEPAL, 1986), mientras un tipo de cambio fijo ocasionaría una causalidad inversa. 
El análisis de precios se extendió desde hace algunas décadas, añadiendo la conducta de las autoridades gubernamentales. En este sentido, se incorpora el supuesto que los consumidores anticipan las subidas de precios, y están informados del comportamiento de las autoridades monetarias y fiscales ${ }^{4}$, que tienen como objetivo estados de baja inflación con producción elevada. En consecuencia, la tasa de inflación está definida por las preferencias de las autoridades gubernamentales y monetarias.

Los modelos de este tipo muestran a la tasa de inflación como dependiente de la evolución de la actividad económica, también desprenden conclusiones útiles para la discusión de políticas de estabilización.

\section{b) Los esquemas de pugna distributiva}

Esta teoría asocia a la inflación con las alzas persistentes en los costos de producción, por tanto, se basa en el supuesto de que los precios de los bienes se determinan directamente a partir de los precios de los factores y los precios relativos (CEPAL, 1986). En los modelos de pugna distributiva, los precios se establecen como resultado de un conflicto social (Kalecki, 1943), que afecta directamente la demanda nominal. Sin embargo, aunque este esquema pueda ser una fuente autónoma del alza de precios, el esquema sólo funciona si es más flexible la política económica.

\section{c) Los modelos de inflación estructural}

CEPAL (1986) advierte desde un principio, que la denominación estructural es meramente ambigua, ya que la formación de precios está vinculada con características específicas de la economía (su composición político-social) y las reglas de política económica. Un déficit fiscal muy

4 La teoría monetaria-fiscal es comúnmente representada por la curva de Phillips ampliada con expectativas: si la demanda global crece, los precios se ajustan inmediatamente, causando que el nivel de producción permanezca constante. Esto implica que los aumentos inesperados en la demanda eleven transitoriamente el nivel de producción esperada, mientras los precios responden menos que proporcionalmente a las variaciones del gasto. 
elevado puede atribuirse a causas estructurales, sin embargo, el estudio de los causales del déficit podría cambiar si este no está relacionado con variables estructurales. Las hipótesis de la inflación estructural se refieren a una clase especifica de modelos, con supuestos muy bien definidos sobre el origen no monetario de la inflación (Sunkel, 1958; Prebisch, 1961). Estas teorías señalan a los aumentos generalizados de precios como provenientes de los desplazamientos de oferta y demanda intersectoriales. El alza de precios de ciertos bienes altamente volátiles tienden a elevar los precios relativos y, por ende, el nivel general de precios. La teoría marca que podría aumentar su intensidad si existen ciertos mecanismos de difusión intersectoriales (CEPAL, 1986) o cambios inducidos en el déficit fiscal.

Las hipótesis sobre formación de precios estructurales se dividen en los bienes de precio flexible (típicamente las materias primas) y los de precios administrados (por las entidades económicas o el gobierno). También, se supone la indexación de los salarios, que ajusta los pagos de los trabajadores mediante el índice de precios. Desde el punto de vista analítico, este tipo de modelos revelan lo difícil que es, bajo un enfoque de permanente equilibrio, explicar la formación de precios $\mathrm{y}$, específicamente, la de los salarios.

\section{Transferencia del precio del petróleo a la inflación en Estados Unidos}

Cuantificar la magnitud y establecer el momento de traspaso de los cambios en el precio del petróleo al Índice de Precios del Consumidor (IPC) en Estados Unidos es crucial para pronosticar la inflación (Conflitti \& Luciani, 2017). Caracterizar este paso es uno de los más importantes porque los precios del petróleo tienden a sufrir grandes fluctuaciones que afectan a la inflación desde el componente energético y no energético.

En teoría, un aumento en los precios del petróleo puede causar un impacto inflacionario. Primero, porque los precios de la energía representan una parte (considerable) de los costos de producción. Segundo, porque podría provocar mayores expectativas de inflación. Tercero, 
porque podría conducir a los trabajadores a percibir un salario más alto para compensar los precios de la energía (Blanchard \& Gali, 2007) y al mismo tiempo podría imitar un shock de oferta adverso si los salarios reales no disminuyen lo suficiente, lo que provocaría un ajuste en el empleo (Bruno \& Sachs, 1985).

Por el contrario, un aumento en los precios del petróleo podría tener un efecto deflacionario como un choque adverso de la demanda, porque los precios más altos de la energía tienden a reducir el ingreso disponible neto y, por lo tanto, el consumo y la inversión (Kilian \& Lewis, 2011).

En nuestra investigación, se toman los precios de la gasolina por los del petróleo. Empíricamente, a lo largo del tiempo la inflación de Estados Unidos ha mantenido cierta consistencia con los movimientos del precio de la gasolina, sin embargo, a partir de diciembre de 2014 se desvincularon ambas variables, mismas que a finales de diciembre de 2018 logran recuperar una misma tendencia (Cf. Gráfica 1).

A pesar de este fenómeno, la correlación entre ambas variables es positiva $y$, a diferencia del precio de otros bienes, su cambio repercute directamente en la determinación de la inflación.

\section{Gráfica 1. USA, Inflación (derecha) y precio de la gasolina regular (dll/gal) (izquierda) (2006-18)}

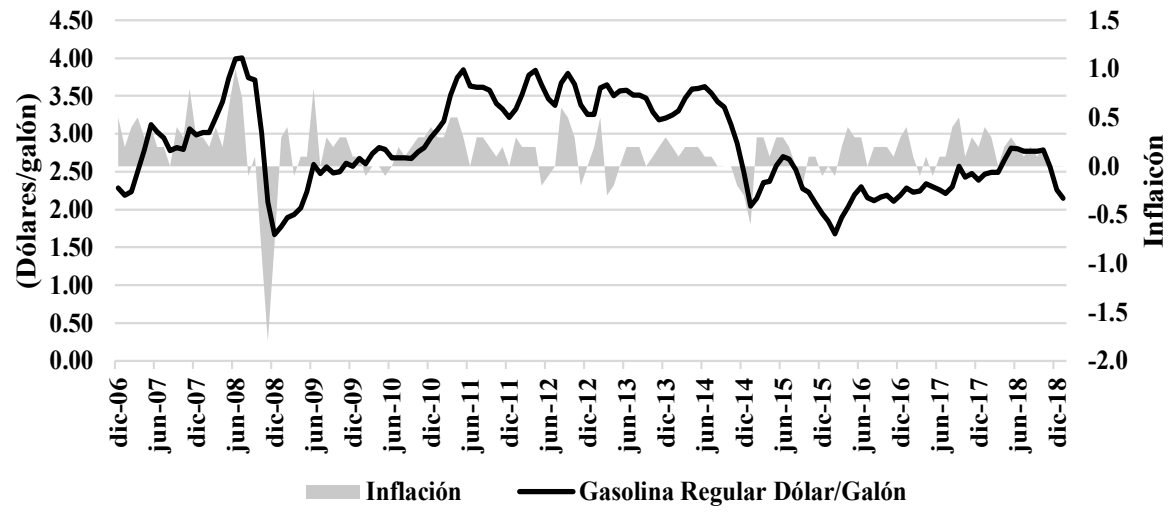

Fuente: EIA (2019). 
Consecuencia de la caída de los precios en la gasolina desde 2014, los ingresos promedio incrementaron gradualmente después de la crisis financiera (2008-2010). Sin embargo, estos se elevan de forma considerable a inicios de 2018 (Cf. Gráfica 2), año donde ambas variables (precio de la gasolina e inflación) vuelven a vincularse positivamente. Estados Unidos desde hace un lustro ha presenciado tasas de desempleo ultra bajas que no se habían visto desde la década de 1950-1970 Cf. Gráfica 3).

\section{Gráfica 2. USA, Ingresos promedio por hora para todas las industrias (2007-19)}

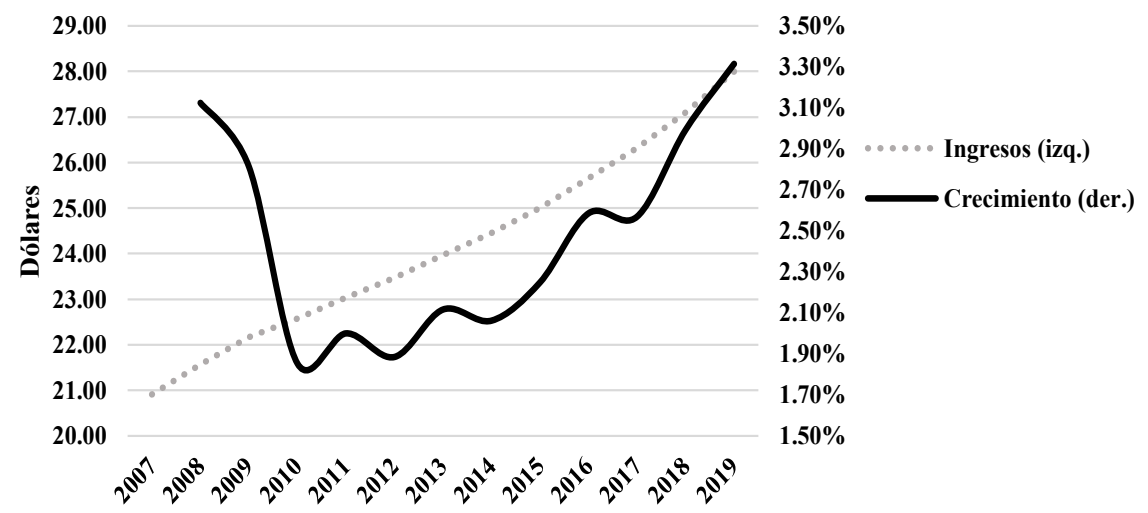

Fuente: BEA (2019).

Gráfica 3. Tasa de desempleo (\% del total de la fuerza laboral) en USA y México (2010-18)

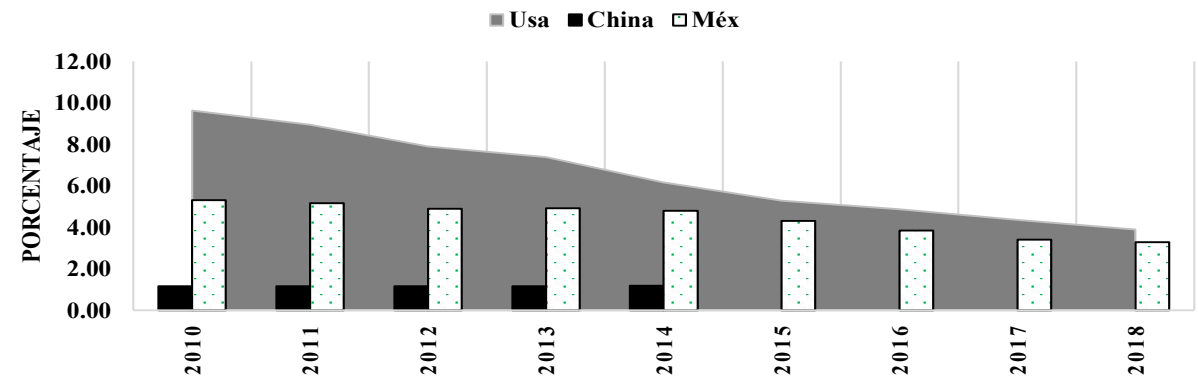

Fuente: ILO (2019). 


\section{La inflación: su impacto en los principales termómetros de la economía}

\subsection{Indicadores estructurales}

Al igual que los precios internacionales, la baja inflación de Estados Unidos ha causado un descenso en el crecimiento económico (Cf. Gráfica 4) (FMI, 2019), mantenimiento niveles de consumo e inversión cercanos a los 13,000 y 3,500 millones de dólares respectivamente (Cf. Gráfica 5).

Gráfica 4. Crecimiento del PIB \% países seleccionados y pronóstico (2020-23)

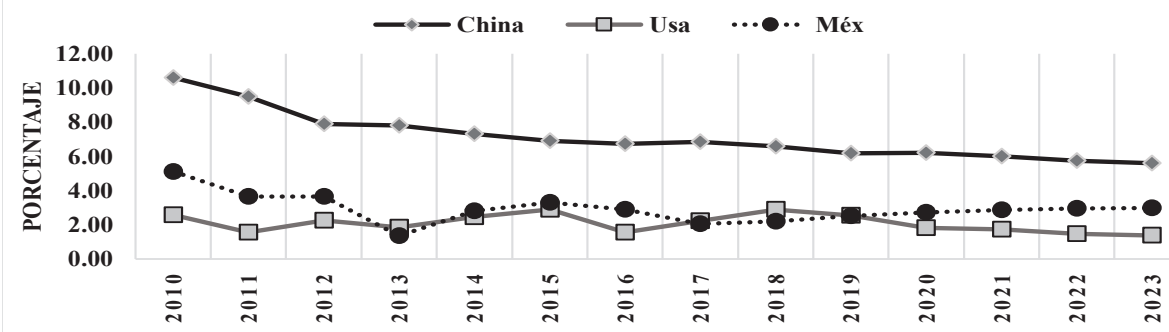

Fuente: FMI (2018).

Gráfica 5. USA, consumo e inversión (eje derecho) privados (2007-19) billones de dólares

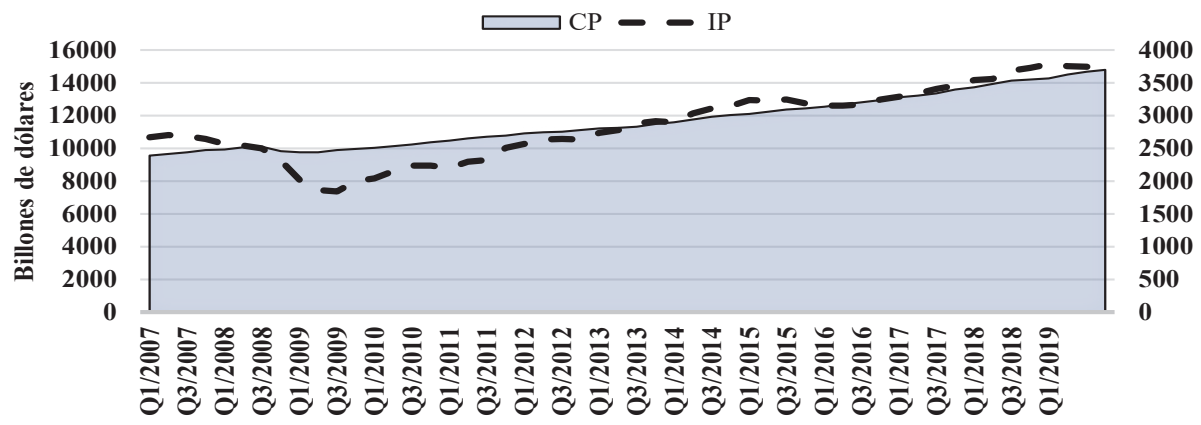

Fuente: BEA (2019). 


\subsection{Indicadores financieros}

Evaluar los principales indicadores del mercado de dinero es importante, ya que permite corroborar la teoría monetaria como un causal de la inflación. Los hechos empíricos exhiben que los niveles de la tasa de interés de corto plazo de EU desde 2010 han permanecido casi en ceros, tendencia que cambió a finales de 2015, elevándose a un máximo de 2.5\% de agosto de 2018 a marzo de 2019 (Cf. Gráfica 6) (FMI, 2019). Considerando la evolución de la economía estadounidense en los últimos meses, los inversionistas volvieron a los bancos, suceso que disminuyó la oferta monetaria y la inflación. También, este hecho es compatible con los años de elevada inflación y tasas de interés ultra bajas. Se incluye la tasa de interés de México para hacer un breve comparativo: desplome desde inicios de 2019 para México y consistencia en Estados Unidos.

\section{Gráfica 6. Tasas de interés a corto plazo: USA (derecha) y México (izquierda) (2018-19)}

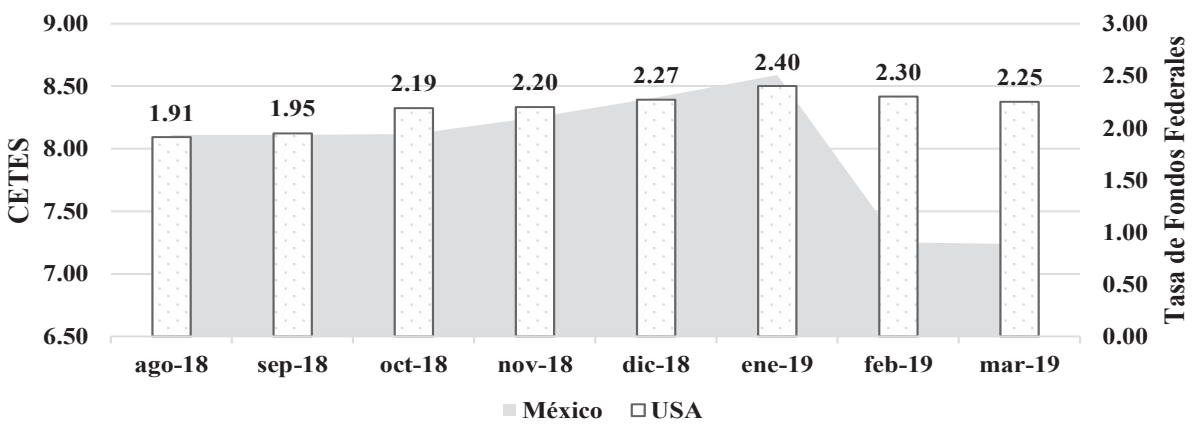

Fuente: FED (2018) OCDE, Banxico (2018).

Caso contrario ocurre para la tasa de interés a largo plazo. Existe una correspondencia con la caída de la tasa de inflación en Estados Unidos, en el mismo periodo (agosto de 2018 a marzo de 2019), por tanto, la tasa de interés de largo plazo no puede ser vista como un determinante inflacionario. 


\section{Gráfica 7. Tasas de interés a largo plazo: USA (derecha) y México (izquierda) (2018-19)}

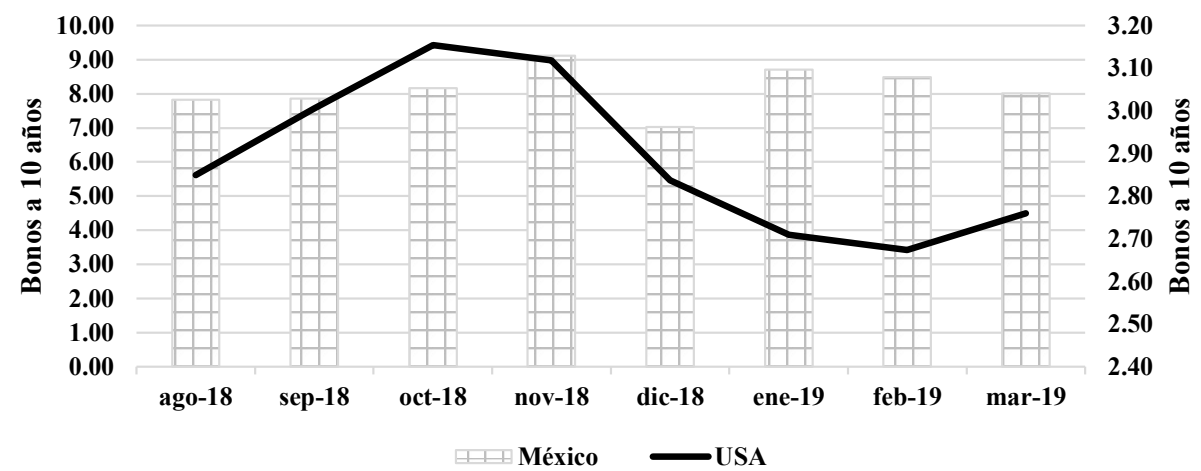

Fuente: FED y BANXICO (2019).

El tipo de cambio respecto a China y México desde 2016 ha permanecido constante pese a la crisis de 2008 , y el poder adquisitivo de su moneda ha incrementado con el tiempo.

Gráfica 8. Comparación del tipo de cambio de China y México, respecto a USA (2007-18)

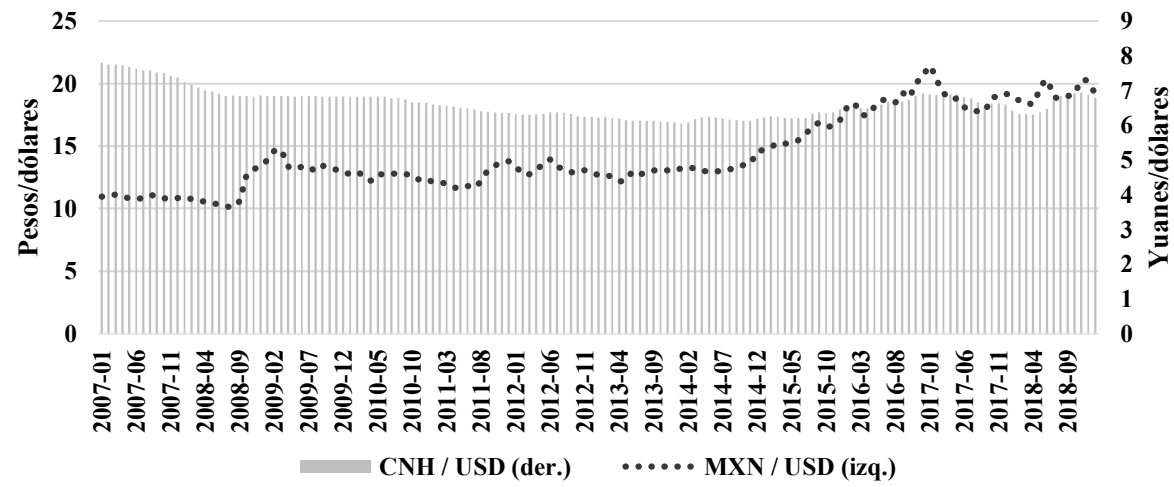

Fuente: FED (2019). 


\section{Modelo ARMAX}

El propósito de esta investigación es determinar el efecto del precio de la gasolina en la inflación bajo el modelo de inflación estructural; teniendo en cuenta que, en efecto, los choques de algunas variables monetarias impactan en el periodo activo de la inflación o como rezagos de un año. Se propone probar dicha hipótesis con la realización de un modelo híbrido que incluya variables explicadas por la misma serie y a la vez, exógenas. El modelo ARMAX considera variables autorregresivas, promedio móvil de la propia serie y además incorpora variables exógenas, por lo cual, el modelo es el más apropiado para cumplir el objetivo de esta investigación ${ }^{5}$.

En el análisis econométrico, se utilizó la gasolina regular como principal referente para vincular sus movimientos con los desequilibrios inflacionarios.

\section{Gráfica 9: USA, precios de gasolina y diesel (USA) según clasificación (2007-18)}

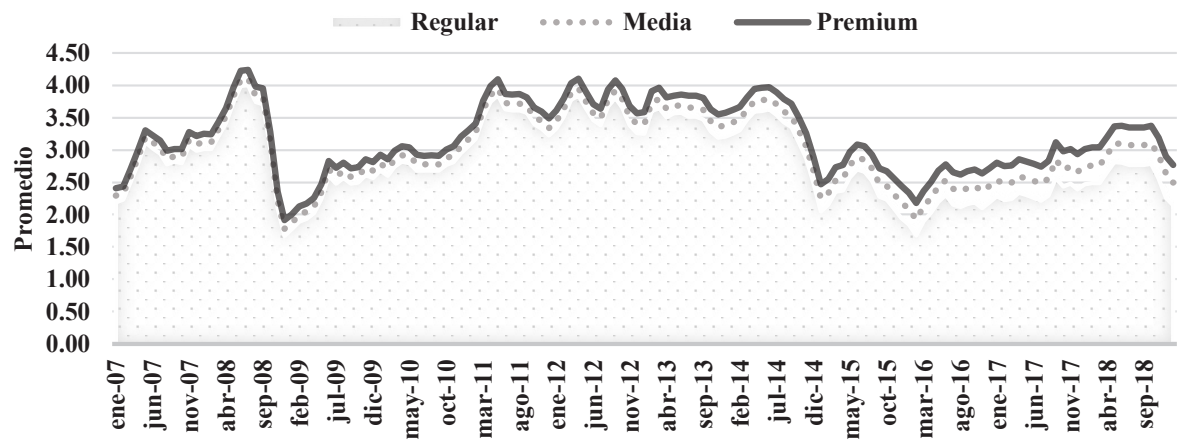

Fuente: EIA (2019).

5 Haciendo una comparación con el uso de otros modelos aplicados tradicionalmente, por ejemplo, se ha sugerido la utilización de un modelo ARMA, pero al hacerlo se incurriría en una contradicción, si bien los resultados muestran un fuerte comportamiento explicado por la misma serie el objetivo del artículo es enfatizar cuál es la explicación de la variación por el efecto de los precios de la gasolina con la inflación y establecer su relación causal. 


\section{Especificaciones del modelo}

Concretamente, se busca estimar un modelo econométrico temporal que compruebe la hipótesis propuesta mediante un modelo ARMAX (Autorregressive Movil Average Exogenous), se realiza una estimación del Índice de Precios al Consumidor (IPC) considerando la variable del precio promedio de la gasolina regular en Estados Unidos (GasReg) en el periodo de enero de 2007 a diciembre de 2018 con periodicidad mensual.

Hipótesis: El precio de la gasolina tiene un efecto significativo en la tasa de variación mensual del IPC.

Se utiliza la variación mensual del IPC con año base 2018 de enero de 2007 a diciembre de 2018:

$$
V M_{-} I P C_{t}=\left(\frac{I N P C_{t}-I N P C_{t-1}}{I N P C_{t-1}}\right) * 100
$$

Como variable exógena se utiliza el precio promedio de la gasolina regular, se consideran las primeras diferencias de la serie:

$$
\begin{aligned}
& d_{-} V M_{-} I P C_{t}=V M_{-} I P C_{t}-V M_{-} I P C_{t-1} \\
& d_{-} \text {GasReg }_{t}=\text { GasReg }_{t}-\text { GasReg }_{t-1}
\end{aligned}
$$

Estadísticos principales, usando las observaciones de enero 2007 a diciembre 2018

\begin{tabular}{|c|c|c|c|c|}
\hline Variable & Media & Mediana & Mínimo & Máximo \\
\hline IPC & 92.759 & 93.819 & 82.802 & 100.71 \\
\hline $\begin{array}{c}\text { Precio } \\
\text { gasolina }\end{array}$ & 2.8489 & 2.7810 & 1.6690 & 4.0020 \\
\hline Variable & Desv. Típica. & C.V. & Asimetría & $\begin{array}{c}\text { Exc. de } \\
\text { curtosis }\end{array}$ \\
\hline IPC & 4.6512 & 0.050143 & -0.22612 & -1.0254 \\
\hline $\begin{array}{c}\text { Precio } \\
\text { gasolina }\end{array}$ & 0.58722 & 0.20612 & 0.069839 & -1.1003 \\
\hline
\end{tabular}




\begin{tabular}{|c|c|c|c|c|}
\hline Variable & Porc. 5\% & Porc. 95\% & Rango IQ & $\begin{array}{c}\text { Observacio- } \\
\text { nes ausentes }\end{array}$ \\
\hline IPC & 84.964 & 100.24 & 7.6475 & 0 \\
\hline $\begin{array}{c}\text { Precio } \\
\text { gasolina }\end{array}$ & 1.9392 & 3.7445 & 1.0520 & 0 \\
\hline
\end{tabular}

Se utiliza un orden AR 3 y 6 , un orden MA 12 no estacional

$$
Y_{t}=\phi_{3} Y_{t-3}+\phi_{6} Y_{t-6}+\beta_{1} X_{1, t}+e_{t}
$$

Donde:

$Y_{t}$ es la variable explicada $d_{-} V M_{-} I P C$ en el tiempo $\mathrm{t}$

$\phi_{3}$ es la estimación del rezago 3 en el proceso autorregresivo

$\phi_{6}$ es la estimación del rezago 6 en el proceso autorregresivo

$\beta_{1}$ es la estimación del cambio en $Y_{t}$ por cambio en $X_{1, t}$

$X_{1, t}$ es la variable explicativa $d \_$GasReg en el tiempo t

$$
Y_{t}=\varepsilon_{t}+\theta_{12} \varepsilon_{t-12}
$$

Donde:

$\varepsilon_{t}$ es el error del modelo

$\theta_{1}$ es la estimación del rezago 12 en el proceso de media móvil

Se obtiene la ecuación

$$
Y_{t}=\phi_{6} Y_{t-6}+\varepsilon_{t}+\theta_{12} \varepsilon_{t-12}+\beta_{1} X_{1, t}+\beta_{2} X_{2, t}
$$

\subsection{Resumen de los resultados obtenidos}

Tabla 1. Estimadores de la regresión obtenidos

\begin{tabular}{|c|c|c|c|}
\hline Coeficientes & Estimados & Desviación típica & Valor $\mathbf{p}$ \\
\hline $\boldsymbol{\phi}_{\mathbf{3}}$ & -0.260477 & 0.0837157 & 0.0019 \\
\hline $\boldsymbol{\phi}_{\mathbf{6}}$ & -0.196348 & 0.0832655 & 0.0184 \\
\hline $\boldsymbol{\theta}_{\mathbf{1 2}}$ & 0.266166 & 0.0744569 & 0.0004 \\
\hline $\boldsymbol{\beta}_{\mathbf{1}}$ & 1.03854 & 0.141961 & $<0.0001$ \\
\hline
\end{tabular}


Los estimadores del modelo confirman una relación positiva con la variación del precio de la gasolina, además de incluir los efectos por rezagos trimestrales de la misma serie, todos significativos.

\section{Pronóstico}

Con base en el modelo estimado se realizó una predicción para el periodo de enero de 2019 a diciembre del mismo año:

\section{Gráfica 10. Proyección/simulación del modelo}

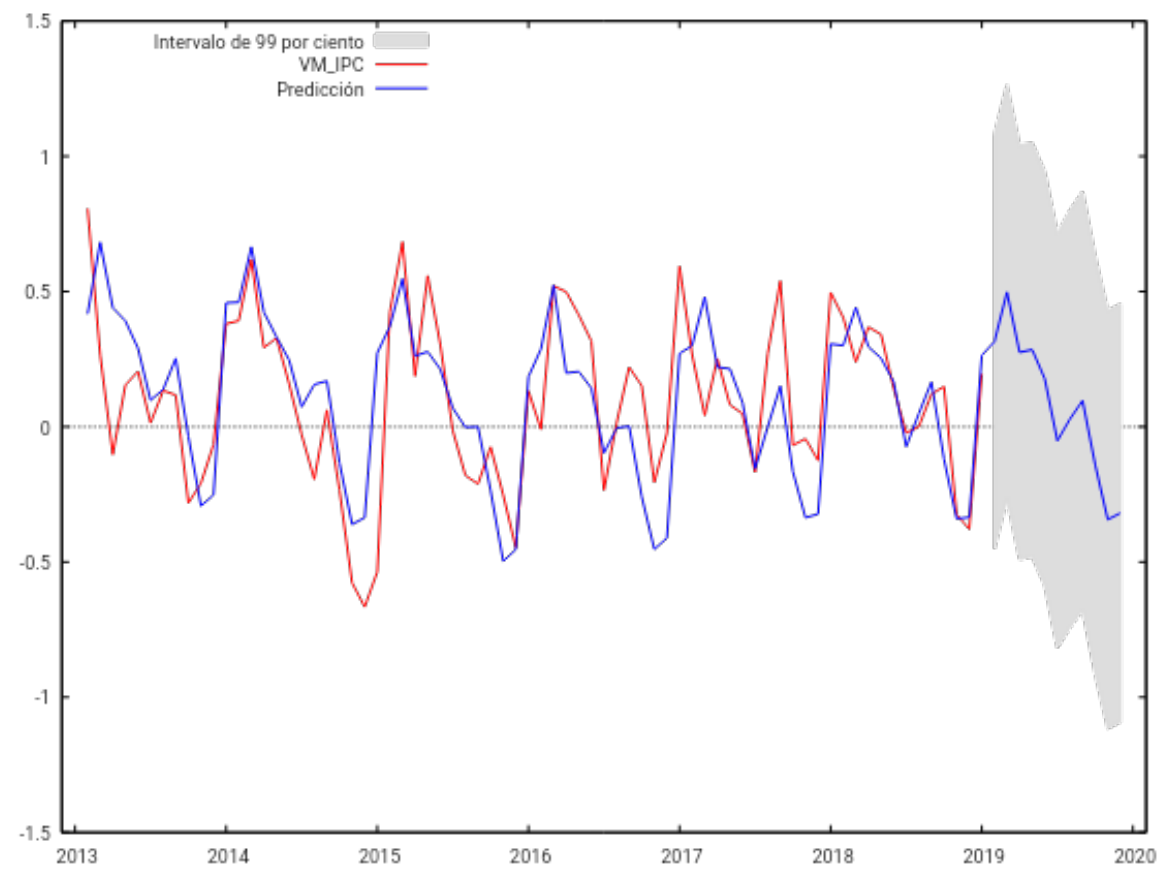

Fuente: EIA (2019).

Como se puede observar en la Gráfica 10, el modelo se ajusta al rango de datos utilizados, además de conservar la misma tendencia para periodos posteriores. Al igual que en las estimaciones previas, los datos futuros dependen de valores rezagados trimestrales de la misma serie y de la fluctuación del precio de la gasolina, por lo que necesita informa- 
ción del precio anterior a su estimación (este comportamiento se verá compensado por la dependencia de la serie propia).

\section{Tabla 2. Valores de la simulación o proyección del modelo}

\begin{tabular}{|l|c|c|c|c|}
\hline \multicolumn{1}{|c|}{$\begin{array}{c}\text { Fecha } \\
(\mathbf{2 0 1 9 )}\end{array}$} & Estimado & $\begin{array}{c}\text { Desviación } \\
\text { típica }\end{array}$ & \multicolumn{2}{c|}{ Intervalo de confianza } \\
\hline Enero & 0.258917 & 0.298249 & -0.325640 & 0.843474 \\
\hline Febrero & 0.314990 & 0.298249 & -0.269567 & 0.899547 \\
\hline Marzo & 0.497749 & 0.298249 & -0.086807 & 1.082306 \\
\hline Abril & 0.275255 & 0.298250 & -0.309303 & 0.859814 \\
\hline Mayo & 0.285255 & 0.298250 & -0.407221 & 0.761896 \\
\hline Junio & 0.177338 & 0.298250 & -0.407221 & 0.761896 \\
\hline Julio & -0.060569 & 0.301132 & -0.650777 & 0.529639 \\
\hline Agosto & 0.028594 & 0.301132 & 0.561614 & 0.618802 \\
\hline Septiembre & 0.097153 & 0.301132 & -0.493056 & 0.687361 \\
\hline Octubre & -0.139138 & 0.301132 & -0.729346 & 0.451070 \\
\hline Noviembre & -0.342946 & 0.301132 & -0.933154 & 0.247263 \\
\hline Diciembre & -0.319248 & 0.301132 & -0.909456 & 0.270960 \\
\hline
\end{tabular}

Para corroborar la cointegración del modelo ARMAX, se analizan los residuos de un modelo por Mínimos Cuadrados Ordinarios y su estacionariedad utilizando las primeras diferencias de las variables a tratar.

$$
d_{-} V M_{-} I P C_{t}=\beta_{1} d_{-} \operatorname{GasReg}_{t}+e_{t}
$$


Gráfica 11. Residuos de la regresión (=d_vm_ipc observada - estimada)

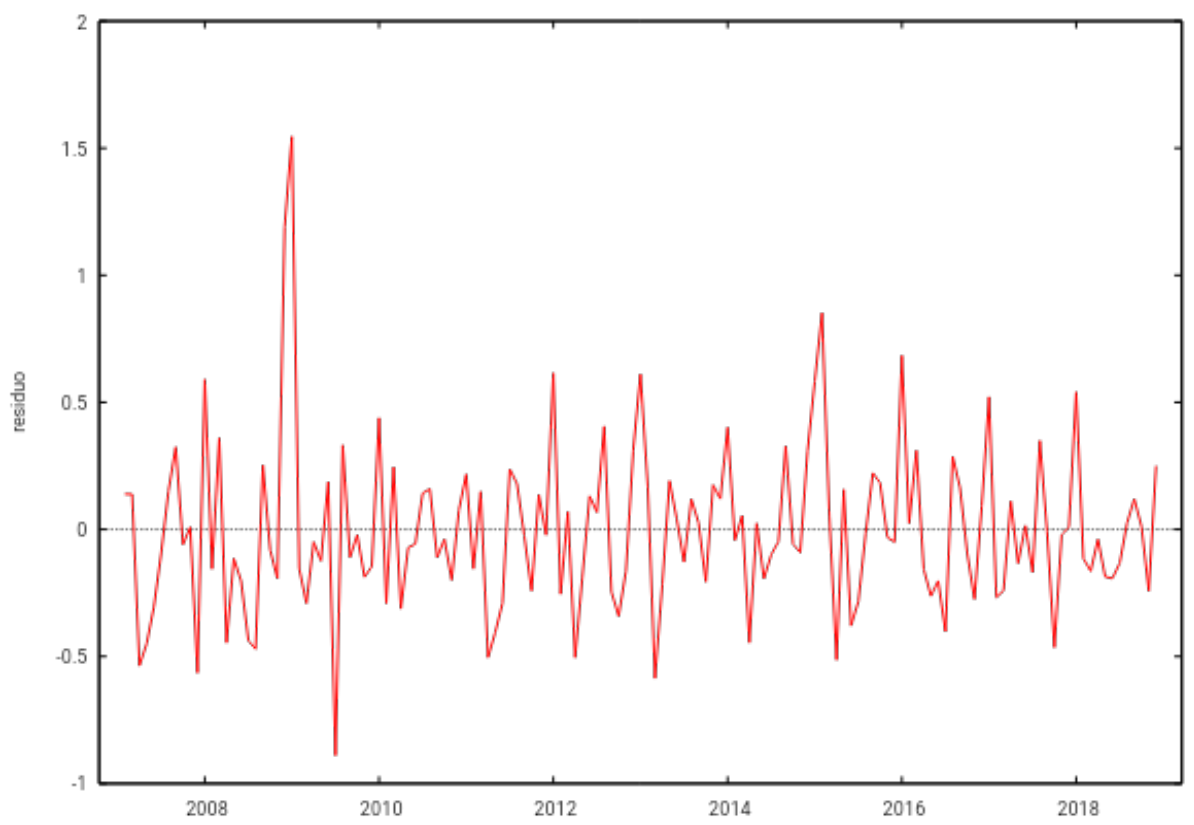

Tabla 3. Pruebas de raíz unitaria

\begin{tabular}{|c|c|c|c|}
\hline \multicolumn{4}{|c|}{ Prueba Dickey-Fuller } \\
\hline & $\begin{array}{c}\text { Contraste sin } \\
\text { constante }\end{array}$ & $\begin{array}{c}\text { Contraste con } \\
\text { constante }\end{array}$ & $\begin{array}{c}\text { Con contraste y } \\
\text { tendencia }\end{array}$ \\
\hline Valor-p & 0.0001 & 0.002973 & 0.1759 \\
\hline \multicolumn{4}{|c|}{ Contraste KPSS } \\
\hline $\begin{array}{c}\text { Estadístico de } \\
\text { contraste }\end{array}$ & 0.085816 \\
\hline
\end{tabular}

Para las pruebas Dickey-Fuller, las 3 rechazan la hipótesis nula (Ho: los residuos no son estacionarios). La prueba KPSS no rechaza marginalmente la hipótesis nula (Ho: la serie es estacionaria). 


\section{Gráfica 12. Correlograma de los residuos de la regresión}

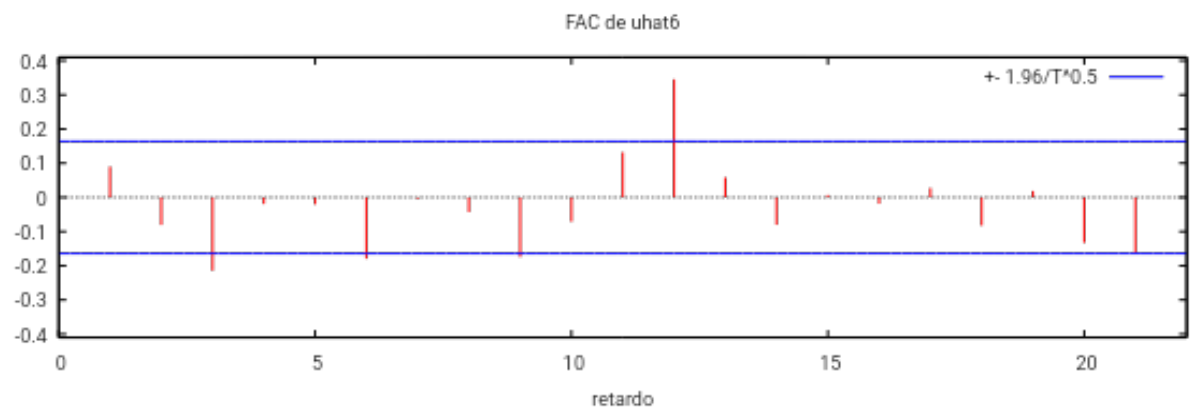

FACP de uhat6

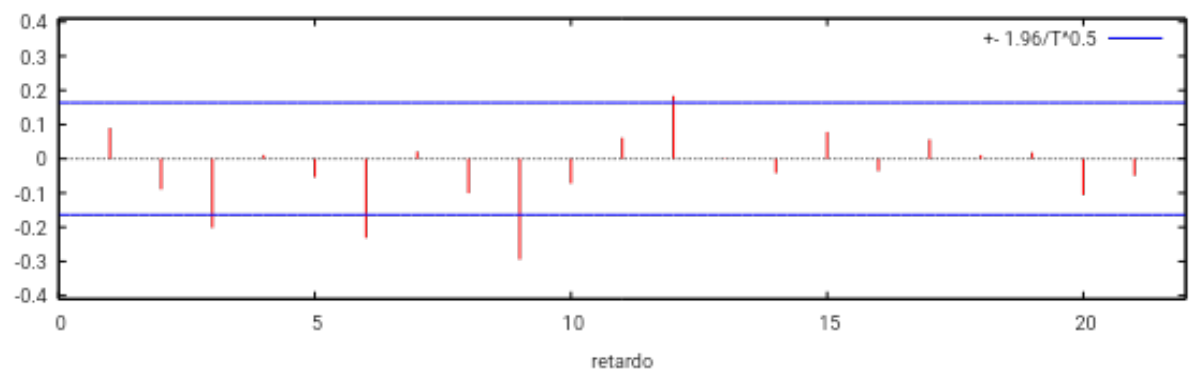

El análisis del correlograma de los residuos, se concluye por utilizar un orden autorregresivo 3 y 6 y un orden media móvil de 12 .

\section{Modelo: ARMAX, usando las observaciones$$
\text { 2007:02-2019:01 }(T=144)
$$ \\ Variable dependiente: d_VM_IPC \\ Desviaciones típicas basadas en el Hessiano}

\begin{tabular}{|c|c|c|c|c|c|}
\hline \multicolumn{2}{|c|}{ Coeficiente } & Desv. Típica & z & \multicolumn{2}{c|}{ valor p } \\
\hline phi_3 & -0.260477 & 0.0837157 & -3.111 & 0.0019 & $* * *$ \\
\hline phi_6 & -0.196348 & 0.0832655 & -2.358 & 0.0184 & $* *$ \\
\hline theta_12 & 0.266166 & 0.0744569 & 3.575 & 0.0004 & $* * *$ \\
\hline d_GasReg & 1.03854 & 0.141961 & 7.316 & $<0.0001$ & $* * *$ \\
\hline
\end{tabular}




\begin{tabular}{|c|c|c|c|}
\hline $\begin{array}{c}\text { Media de la vble. } \\
\text { dep. }\end{array}$ & -0.000601 & D.T. de la vble. dep. & 0.381345 \\
\hline $\begin{array}{c}\text { Media de innovacio- } \\
\text { nes }\end{array}$ & -0.002251 & D.T. innovaciones & 0.301464 \\
\hline R-cuadrado & 0.372798 & R-cuadrado corregido & 0.359358 \\
\hline Log-verosimilitud & -32.31078 & Criterio de Akaike & 74.62156 \\
\hline Criterio de Schwarz & 89.47063 & Crit. de Hannan-Quinn & 80.65539 \\
\hline
\end{tabular}

\section{Gráfica 13. Supuesto de autocorrelación de los rezagos por medio del correlograma de los residuos}

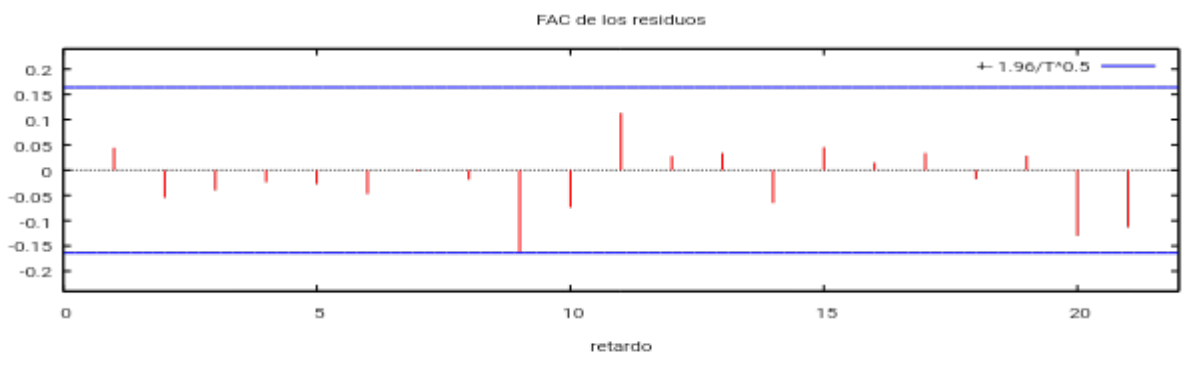

FACP de los residuos

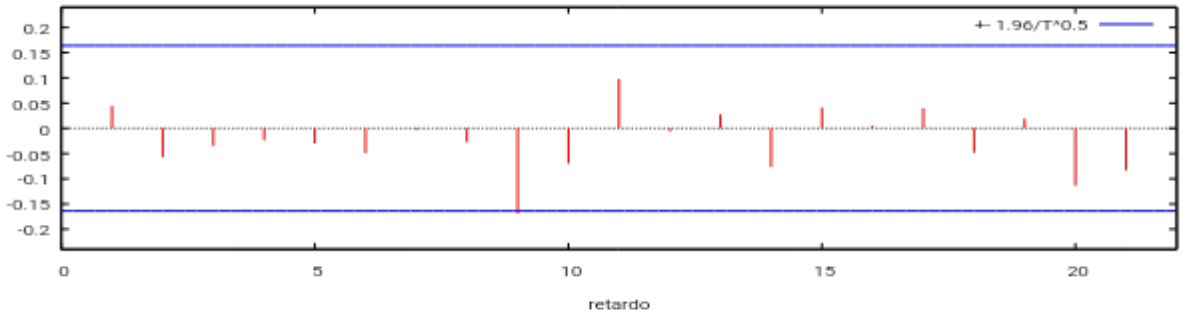

\subsection{Comentarios del modelo estimado}

La estimación realizada no garantiza su pronóstico para periodos posteriores, solamente para el lapso estudiado. El modelo revela la necesidad de un análisis más riguroso para el efecto causal de la inflación con el precio de la gasolina y así realizar una proyección más certera o a largo plazo, además de servir como un antecedente para la comprensión de la determinación de la inflación bajo un enfoque estructural. El hallazgo más importante del modelo es que, en efecto, el precio de la gasolina es un causal imprescindible en la determinación de la inflación. 


\section{Conclusiones}

Existen innumerables debates acerca de los causales de la inflación, sin embargo, todos están sujetos a deducciones que pueden resultar demasiado simplistas o rigurosas para determinar el porqué de un proceso inflacionario. Lo verdaderamente cierto de la evidencia empírica de Estados Unidos es que, en efecto, existen causales monetarios y estructurales que repercuten directamente en la inflación. Este es el caso de la gasolina regular que, a través de la inflación subyacente, tiene un efecto significativo en la formación de precios. Se debe seguir desarrollando la investigación de los causales estructurales e intentar llegar a una conciliación entre ambas teorías, actualmente válidas.

\section{Bibliografía}

Blanchard, O., \& Gali, J. (2007). "The Macroeconomic Effects of Oil Shocks: Why are the 2000s So Different from the 1970s?", International Dimensions of Monetary Policy, 373-421.

Bruno, M., \& Sachs, J. (1985). Economics of Worldwide Stagflation, Cambridge: Harvard University Press.

CEPAL. (1986). Tres ensayos sobre inflación y políticas de estabilización, Oficina de CEPAL en Buenos Aires, Estudios e informes de la CEPAL.

Conflitti, C., \& Luciani, M. (2017). Oil price pass-through into core in, Federal Reserve.

FMI. (2019). Perspectivas de la economía mundial (WEO), actualización de las proyecciones centrales, Washington.

Friedman, M. (1968). "The role of monetary policy", The American Economic Review, p. 17.

Gutiérrez Rodríguez, Roberto (2019). “México 2016-2018: ¿inicio de una nueva fase de desarreglo inflacionario?", ECONOMÍAunam, Vol. 16, No. 47, mayo-agosto.

Heath, J. (2012). Lo que indican los indicadore, INEGI. 
Kilian, L., \& Lewis, L. (2011). "Does the Fed respond to oil price shocks?", The Economic, pp. 1047-1072.

Prebisch, R. (1961). Desarrollo económico, planeamiento y cooperación internacional, Santiago de Chile, CEPAL.

Sunkel, O. (1958). "La inflación chilena: un enfoque heterodoxo", $E l$ Trimestre Económico. 